\title{
Impacto das Parametrizações de Microfísica na Previsão de Precipitação utilizando Assimilação de Dados de Radar
}

\author{
Rute Costa Ferreira $^{1}$ (D), Mario Paulo Alves Júnior ${ }^{2}$ (D), Éder Paulo Vendrasco ${ }^{1}$ (D), \\ José Antônio Aravéquia $^{1}$ (D), Luciano Ritter Nolasco Junior ${ }^{1}$ (D), Thiago Souza Biscaro ${ }^{1}$ \\ ${ }^{1}$ Centro de Previsão do Tempo e Estudos Climáticos, Instituto Nacional de Pesquisas Espaciais, \\ Cachoeira Paulista, SP, Brasil. \\ ${ }^{2}$ Instituto de Controle do Espaço Aéreo, São José dos Campos, SP, Brasil.
}

Recebido em: 17 de Julho de 2019 - Aceito em: 22 de Novembro de 2019

\begin{abstract}
Resumo
Trabalhos recentes mostram que a assimilação de dados melhora a eficácia dos modelos de previsão de tempo, contudo o impacto da assimilação dos dados de radar é pouco quantificado com relação às parametrizações físicas do modelo, especialmente de microfísica. O objetivo deste trabalho é estudar o impacto do uso de dados de radar com diferentes parametrizações de microfísica do modelo Weather Research and Forecasting (WRF) com seu sistema de assimilação de dados (WRFDA-3DVAR) para casos de precipitação intensa. Foram selecionados três eventos de precipitação em 2014, com área de estudo abrangendo o oeste da região sul do Brasil e sudeste do Paraguai. Desta forma, são avaliados nove esquemas de parametrizações de microfísica com assimilação de dados convencionais e de radar, para determinar qual representa de forma mais adequada a precipitação e refletividade nas previsões de curto prazo, além de determinar o impacto relativo entre as mudanças de microfísica e a assimilação de dados convencionais e de radar. A comparação realizada através da métrica estatística Fractional Skill Score (FSS) mostra o impacto positivo da assimilação de dados do radar foi na média de até $20 \%$ no FSS, enquanto que o impacto positivo entre as opções de microfísica atingiu $70 \%$.
\end{abstract}

Palavras-chave: parametrização de microfísica, assimilação de dados de radar, WRF e WRFDA.

\section{The Impact of Microphysics Parameterization on Precipitation Forecast Using Radar Data Assimilation}

\begin{abstract}
Recent studies show that data assimilation improves the efficiency of weather forecast models, however, it is not properly quantified the impacts of radar data assimilation related to the physical model parameterizations, especially the microphysics. The goal of this study was to study the impact of the use of radar data with different microphysics parameterizations of the Weather Research and Forecasting (WRF) model with its data assimilation system (WRFDA3DVAR) for cases of intense precipitation. The study area covers the South-western Brazil and Southeastern Paraguay. The simulations were done for three cases in 2014. The comparison is performed through the statistical metrics Fractional Skill Score (FSS) and Local Root Mean Square Error (LRMSE). Different microphysics parameterizations were tested when assimilating conventional and radar data for three events. Thus, we evaluated nine microphysical parameterizations in order to determine which one provides the most realistic short-term forecasts of meteorological fields over the radar coverage, as well as the relative impact of different microphysical parameterization and the assimilation of conventional and radar data. The positive impact of the radar data assimilation was in the average up to $20 \%$ in the FSS, while the positive impact among the microphysics options reached $70 \%$ in the FSS.
\end{abstract}

Keywords: microphysics parameterization, radar data assimilation, WRF and WRFDA.

Autor de correspondência: Mario Paulo Alves Júnior, mpajpdx@gmail.com. 


\section{Introdução}

A ocorrência de precipitação intensa possui grande impacto na sociedade e é o fenômeno causador de enchentes, inundações e alagamentos. No entanto, a precipitação é uma das variáveis mais difíceis de se prever visto que os modelos numéricos ainda falham em organizar a convecção de forma correta no tempo e no espaço (Stensrud et al., 2000). A evolução dos supercomputadores e também a forma que as observações meteorológicas são obtidas, contribuem cada vez mais para a realização de uma melhor previsão do tempo, possibilitando que os modelos numéricos efetuem previsões mais detalhadas. Sendo tais previsões fundamentais para a prever condições adversas de tempo que são de suma importância, desde segurança a vida até a minimização de prejuízos materiais.

A Previsão Numérica de Tempo (PNT) é um problema de condição inicial, no qual uma maior precisão das informações iniciais poderá levar a uma solução mais próxima da realidade. Portanto, a assimilação de dados é uma ferramenta que é utilizada para aproximar de forma mais acurada a condição inicial utilizada nos modelos numéricos para o verdadeiro estado da atmosfera, sendo chamada de análise final a melhor representação possível alcançada na resolução do problema. Porém, deve-se ressaltar que o verdadeiro estado da atmosfera não será representado, devido às imperfeições do modelo, aos erros ao definir a representatividade das observações, às imprecisões dos instrumentos e ao número insuficiente de observações por toda a atmosfera.

Diversos processos são parametrizados dentro de um modelo de PNT, onde estes esquemas de parametrização representam apenas os aspectos essenciais do processo físico envolvido, dada a dificuldade em reproduzir corretamente todos processos detalhadamente por meio de relações simplificadas e limitadas para uma variedade de condições ambientais. Parametrizações são geralmente desenvolvidas para escalas horizontais pré-estabelecidas, ou seja, apenas aqueles aspectos do sistema físico que não são resolvidos pelo modelo precisam ser parametrizados. Desta forma, as parametrizações devem ser relacionadas com a resolução do modelo, onde o aumento do poder computacional e, consequentemente, da resolução de grade, o próprio modelo começa a resolver parcialmente alguns processos parametrizados (Houze, 2014). O desempenho de algumas parametrizações depende também dos processos meteorológicos que ocorrem numa determinada época do ano e da região geográfica. Por exemplo, algumas parametrizações convectivas são mais apropriadas para latitudes médias, enquanto outras têm melhor desempenho nos trópicos. Um problema relacionado aos modelos globais, é que estes utilizam as mesmas parametrizações para todas as áreas geográficas, eliminando a opção de escolher aquelas mais adequadas para uma determinada região (Warner, 2010). A mesma dificuldade é encontrada em modelos regionais para áreas extensas, como é o caso do modelo WRF operacional do Centro de Previsão de Tempo e Estudos Climáticos (CPTEC) que cobre toda a América do Sul, com regiões climáticas bem distintas.

No caso da precipitação, resultado de um dos processos físicos mais complexos da atmosfera devido a sua variabilidade no tempo e no espaço, as parametrizações responsáveis por sua representação são as de cumulus e microfísica. A contribuição de cada uma das parametrizações no total de precipitação varia de acordo com a resolução do modelo. Na resolução de $2 \mathrm{~km}$, como é o caso deste trabalho, apenas a parametrização de microfísica é considerada. A microfísica de nuvens engloba todos os processos físicos que ocorrem na escala de gotículas de nuvem e dos hidrometeoros, o que resulta no tipo de precipitação - seja convectiva ou estratiforme - na intensidade e na distribuição espacial que será prevista (Warner, 2010).

De acordo com Wang (2013), os esquemas de microfísica são usados para simular os efeitos de transporte, mudança nos estados físico e termodinâmico do conjunto de hidrometeoros líquidos ou congelados presentes nas nuvens. Independente do grau de complexidade, o papel do esquema de microfísica é simular a dinâmica do conjunto de hidrometeoros como resposta aos movimentos do ar e fornecendo retorno ao ambiente com base no comportamento das partículas individuais de cada hidrometeoro. Sendo assim, os processos de microfísica se relacionam com as parametrizações físicas de radiação, a partir dos efeitos de nuvens; parametrização cumulus a partir do entranhamento; e com parametrizações da superfície na forma de precipitação.

Com o objetivo de obter condições iniciais mais realistas da atmosfera, a assimilação de dados de radares meteorológicos é uma importante ferramenta, uma vez que estes dados possuem alta resolução espacial e temporal. Entretanto, assim como medições de satélites, as medições de radares têm relação não linear com variáveis do estado do modelo, o que torna seu uso direto um grande desafio. Ainda assim, Aravéquia et al. (2011) e Zhang et al. (2013) testaram a assimilação de dados de radiância para melhorar os prognósticos do modelo e foi notado que a assimilação por conjunto de radiâncias que são afetadas pela precipitação, melhoraram a qualidade de precipitação analisada em termos de localização e da intensidade de chuva acumulada. Por outro lado, a assimilação de dados também pode ser utilizada para estimar as variáveis que não são observadas diretamente, mas que são importantes para diversas aplicações e também para interpolar e extrapolar os dados de sensoriamento remoto conforme Reichle (2008). A inclusão de dados de radar nos modelos pode melhorar significativamente os resultados nas primeiras horas de previsão devido à redução do spinup do modelo 
(Gao et al. 2007), o que torna esta ferramenta muito importante para previsão de curto prazo.

Segundo Aksoy et al. (2009), observações com radar Doppler de refletividade e velocidade radial, têm sido utilizadas em sistemas complexos de assimilação de dados com o intuito de melhorar as condições iniciais de modelos de alta resolução. Porém a inclusão de grande quantidade de dados em altíssima resolução, fornecidos pelo radar, podem alterar o balanço da análise entre as variáveis dinâmicas e microfísicas, gerando convecção espúria.

Além disso, Wang et al. (2013) relataram o baixo impacto da assimilação direta da refletividade em seus resultados encontrados Xiao et al. (2007), por meio de experimentos com assimilação de dados de radar. Com base nisso, Wang et al. (2013) revisaram o método de assimilação de refletividade e apontaram problemas no uso da linearização da relação entre refletividade e a razão de mistura de água de chuva (Z-qr) no operador observação. Desta forma, os autores desenvolveram uma nova metodologia para a assimilação de refletividade, na qual a razão de mistura da água de chuva derivada da refletividade é assimilada, ao invés da assimilação direta da refletividade. Assim, Wang et al. (2013) mostraram que a assimilação da refletividade de forma indireta melhora significativamente a previsão de precipitação de curto prazo (até 7 h) de forma que num determinado caso na China, o método utilizado aumentou a umidade, a água da chuva e a energia potencial convectiva disponível na região de convecção. Como resultado, a análise previu bem o desenvolvimento do sistema convectivo e melhorou a previsão da localização e intensidade da precipitação. Estes resultados foram confirmados por Vendrasco et al. (2013) e Vendrasco (2015) no Brasil. Vendrasco (2015) usou a assimilação de dados de radar direta e indireta para melhorar a previsão de precipitação em curto prazo no Vale do Paraíba. Foi observado que a assimilação indireta dos dados de radar reproduz melhor o posicionamento da precipitação e sua intensidade, enquanto que a assimilação direta de dados da refletividade melhora o posicionamento dos sistemas, mas não reproduz a intensidade de precipitação observada pelo próprio radar. No presente trabalho será empregada a mesma metodologia de assimilação indireta de refletividade.

Devido à dificuldade em medir as taxas de produção de hidrometeoros associados a processos microfísicos individuais na natureza, avaliar o desempenho desses esquemas em termos da modelagem atmosférica e medições de radar pode fornecer informações sobre a eficácia dos esquemas na simulação de sistemas precipitantes. Diante deste cenário, o presente trabalho mostra a importância da escolha da parametrização de microfísica utilizada em simulações com foco na assimilação de dados de radar (ADR). Assim, o objetivo deste trabalho é avaliar o impacto da ADR na previsão de curto prazo e indicar as parametrizações de microfísica mais eficazes quando em- pregada a ADR. São mostrados resultados para performance do modelo WRF com assimilação de dados meteorológicos convencionais e dados de radar, e o impacto que a escolha da microfísica tem sobre a previsão de até $12 \mathrm{~h}$ de precipitação.

Deste modo, na seção 2 são mostrados a área de estudo, dados utilizados, configuração do modelo, metodologia utilizada na assimilação de dados e parametrizações microfísicas avaliadas. Na seção 3 são mostrados os resultados estatísticos da assimilação para a previsão de precipitação para os três casos selecionados e um comparativo da refletividade gerada pelo modelo. As conclusões e sugestões de trabalhos futuros são mostradas na seção 4 .

\section{Materiais e Métodos}

Para atingir o objetivo proposto, foram selecionados três sistemas convectivos de mesoescala (SCM) que ocorreram na Região Sul do Brasil (Fig. 1), onde foram escolhidos de acordo com a diferença da intensidade da precipitação e dos fenômenos meteorológicos causados (Tabela 1).

A disponibilidade dos dados de radar e de dados convencionais sobre a região de estudo também foram fatores relevantes nas escolhas dos casos, onde são selecionados três radares para assimilação, sendo: Assunção (Paraguai), Cascavel (Brasil) e Teixeira Soares (Brasil). A presença destes três radares na área de estudo deste trabalho permite realizar um monitoramento constante dos fenômenos meteorológicos, auxiliando na realização de previsões de tempo de curto prazo, emissão de alertas e assim tentando reduzir ao máximo as perdas e danos principalmente nos estados do Paraná, Santa Catarina e no sul do Paraguai. Além disso, os radares auxiliam na navegação aérea da região, que possui um fluxo de aeronaves intenso e rotas aéreas importantes.

Os radares de Cascavel (CAS) e Teixeira Soares (TXS) são operados pelo Sistema Meteorológico do Paraná (SIMEPAR), enquanto que o de Assunção (ASU) é operado pela Dirección Nacional de Aeronáutica Civil (DINAC). O CAS e o TXS são radares de banda S, com abertura de feixe de $1.0^{\circ}$ e com 15 e 13 elevações, respectivamente. O CAS possui um alcance de 240 e $480 \mathrm{~km}$, operando com resolução máxima de $125 \mathrm{~m}$, enquanto que o TXS possui alcance de $240 \mathrm{~km}$ e resolução de $250 \mathrm{~m}$. O ASU é um radar banda $\mathrm{C}$, com abertura do feixe de $1.0^{\circ} \mathrm{e}$ 11 elevações, alcançando $250 \mathrm{~km}$ com resolução de $250 \mathrm{~m}$.

Apesar dos dados de radares serem muito importantes para o monitoramento meteorológico e também para a assimilação de dados, é importante ressaltar que é um dado no qual a qualidade é extremamente sensível à manutenção e calibração do mesmo. Desta forma, é importante destacar que não houve nenhum controle de 


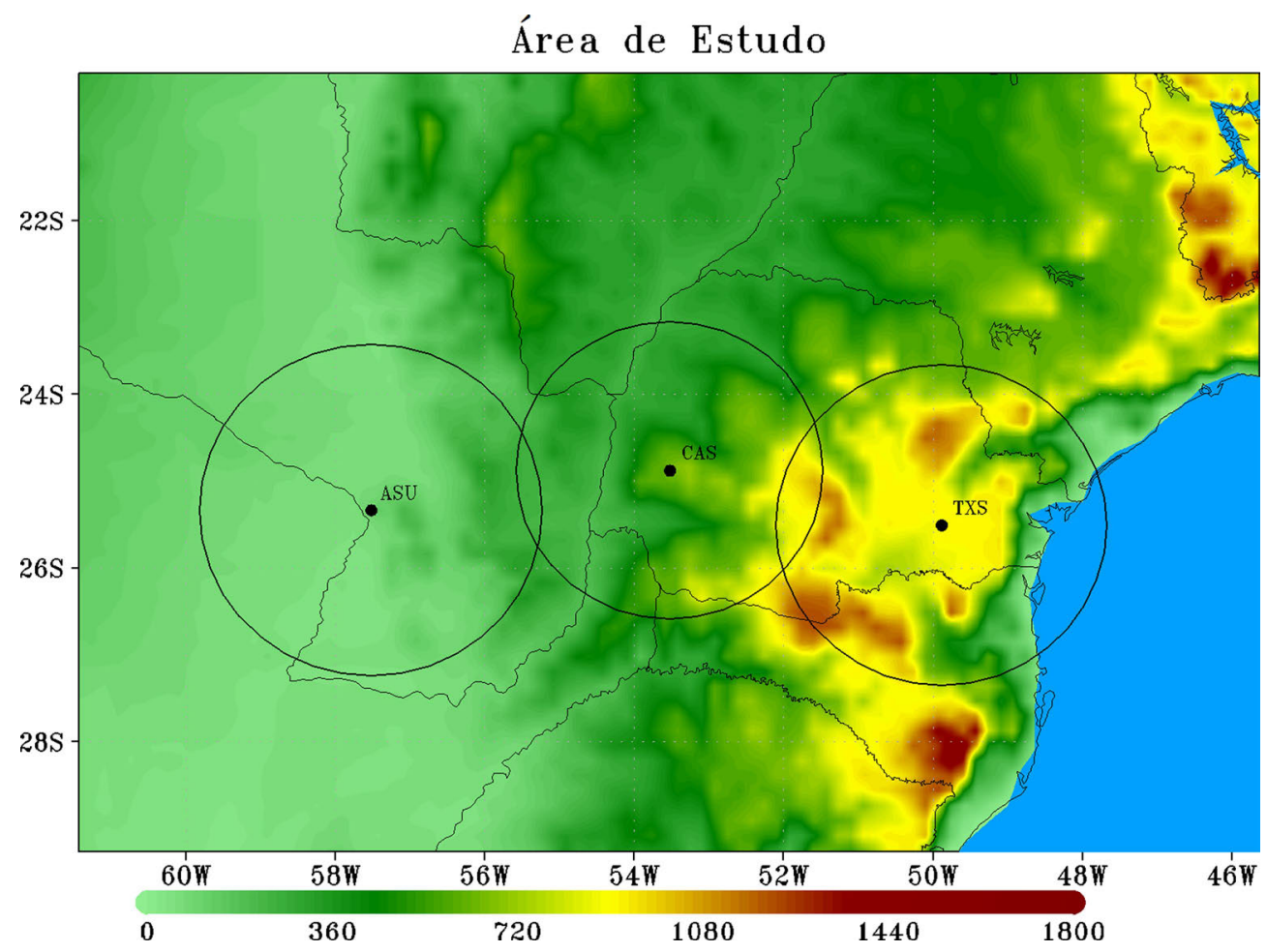

Figura 1 - Posição aproximada dos radares de Assunção (ASU), Cascavel (CAS) e Teixeira Soares (TXS) e seu relevo em metros.

Tabela 1 - Datas dos eventos escolhidos para o estudo e os fenômenos meteorológicos causados.

\begin{tabular}{lcc}
\hline Evento & Data & Fenômeno Meteorológico \\
\hline 1 & $30 / 10 / 2014$ & Convecção local e isolada \\
2 & $07 / 11 / 2014$ & Convecção intensa associada a sistema frontal \\
3 & $13 / 12 / 2014$ & Convecção local e isolada \\
\hline
\end{tabular}

qualidade adicional ao controle já empregado localmente pelo próprio sistema do respectivo radar.

A partir dos dados de refletividade e velocidade radial do vento, foram gerados PPIs (Plan Position Indicator) com resolução de $500 \mathrm{~m}$ para os três radares. Os PPIs foram convertidos para o formato Little-R que é o formato utilizado na assimilação destes dados pelo sistema de assimilação. Os dados assimilados foram aqueles mais próximos da hora exata do ciclo considerado, com máxima diferença de 10 minutos.

Como condição inicial e de contorno para o primeiro ciclo de assimilação (background), são utilizados os dados de análise do modelo Global Forecast System (GFS), disponibilizados pelo National Centers for Environmental Prediction (NCEP), com resolução espacial de $0.5^{\circ} \times 0.5^{\circ}$ e resolução temporal de $6 \mathrm{~h}$. Os dados observacionais que serão assimilados são compostos por observações de superfícies (SYNOP, METAR, boias oceânicas, entre outros) e de altos níveis (radiossondas e aeronaves) que são provenientes do Global Telecommunications System (GTS).

\subsection{Modelagem e assimilação de dados}

$\mathrm{O}$ modelo utilizado foi o Weather Research and Forecast (WRF), que é um modelo regional, com o núcleo dinâmico Advanced Research Weather (ARW) e o sistema de assimilação usado foi o WRF Data Assimilation (WRFDA) com assimilação variacional de dados em 3 dimensões (3D-Var). A versão utilizada do WRF e do WRFDA foi a 3.7.1, sendo possível realizar a assimilação indireta dos dados radar. O modelo foi configurado com uma grade de resolução horizontal de $2 \times 2 \mathrm{~km}$, centralizada nas coordenadas do radar de CAS (Fig. 1), com 800 pontos de longitude e 500 pontos de latitude e 45 níveis na vertical.

As parametrizações físicas utilizadas foram: para radiação de onda longa foi empregada a Rapid Radiative Transfer Model (Mlawer et al. 1997), para a radiação de onda curta foi a Dudhia Shortwave Scheme (Dudhia, 1989), para a camada limite planetária foi a Yonsei University Scheme (YSU) (Hong et al. 2006), para a camada superficial foi o esquema de difusão térmica de 5 camadas 
(Dudhia, 1996) e para cumulus foi desligada, tornando a convecção explícita no modelo, ou seja, proveniente totalmente da microfísica. As parametrizações físicas acima foram mantidas para todos os experimentos, com variação apenas nas opções de parametrizações de microfísica de nuvens, conforme é descrito adiante.

O modelo WRF possui 23 parametrizações de microfísica disponíveis. Dentre estas, foram escolhidas 9 parametrizações para serem estudadas: Kessler e Lin por serem as mais simples; Ferrier por ser amplamente utilizada na literatura e de forma operacional; WSM3, WSM5 e WSM6 foram escolhidas de forma a testar a eficácia da quantidade de hidrometeoros considerada em parametrizações de um momento (single moment); as parametrizações Morrison e WDM6, que são uma evolução na quantidade de hidrometeoros e são dois momentos (double moment); Thompson por ser uma parametrização 1-M mais recente. Maiores detalhes sobre as parametrizações são apresentadas na Tabela 2. Note que todas as microfísicas escolhidas para comparação são ao menos capazes de representar além do vapor $(\mathrm{Qv})$, razão de mistura de água de chuva (Qr) e de água de nuvem (Qc). Maiores informações sobre todos os processos e equacionamentos envolvidos em cada esquema de microfísica podem ser encontrados a partir da referência contida na coluna 3 da Tabela 2.

Os dados de refletividade e velocidade radial do radar são assimilados no sistema WRFDA na forma de razão de mistura de água de chuva (Qr) e componentes do vento $(\mathrm{u}, \mathrm{v})$ respectivamente. Toda a formulação e descri-

Tabela 2 - Opções de parametrizações de microfísica de nuvens testadas no trabalho, no qual mp_ph representa o número da opção de acordo o modelo WRF (Skamarock et al. 2008) v.3.7. As variáveis representadas por cada esquema de parametrização microfísica são: razão de mistura de água de chuva (Qr), razão de mistura de água de nuvem (Qc), razão de mistura de neve (Qs), razão de mistura de gelo (Qi), razão de mistura de graupel $(\mathrm{Qg})$, razão de mistura de granizo $(\mathrm{Qh})$.

\begin{tabular}{|c|c|c|c|}
\hline mp_ph & Esquema & Referência & Variáveis \\
\hline 1 & Kessler & Kessler (1969) & Qv Qc Qr \\
\hline 2 & Lin (Purdue) & Lin et al. (1983) & $\begin{array}{c}\text { Qv Qc Qr Qi Qs } \\
\text { Qg }\end{array}$ \\
\hline 3 & WSM3 & Hong et al.. (2004) & Qv Qc Qr \\
\hline 4 & WSM5 & Hong et al. (2004) & Qv Qc Qr Qi Qs \\
\hline 5 & $\begin{array}{l}\text { Ferrier (Nova } \\
\text { Eta) }\end{array}$ & Rogers et al. (2001) & Qv Qc Qr Qs \\
\hline 6 & WSM6 & Hong e Lim (2006) & $\begin{array}{c}\text { Qv Qc Qr Qi Qs } \\
\text { Qg }\end{array}$ \\
\hline 8 & Thompson & $\begin{array}{l}\text { Thompson et al. } \\
\text { (2008) }\end{array}$ & $\begin{array}{c}\text { Qv Qc Qr Qi Qs } \\
\text { Qg }\end{array}$ \\
\hline 10 & Morrison 2-mom & Morrison et al. (2009) & $\begin{array}{c}\text { Qv Qc Qr Qi Qs } \\
\text { Qg }\end{array}$ \\
\hline 16 & WDM6 & Lim e Hong (2010) & $\begin{array}{c}\text { Qv Qc Qr Qi Qs } \\
\text { Qg }\end{array}$ \\
\hline
\end{tabular}

Fonte: Adaptado de USER GUIDE WRF 3.7. ção do equacionamento do operadores observação podem ser encontrados em Sun e Crook (1997); Wang et al. (2013); Vendrasco el al., (2016); Ferreira et al. (2017).

A configuração das rodadas iniciais para cada evento inclui ciclos de $6 \mathrm{~h}$, sendo as 9 primeiras rodadas, uma referente a cada parametrização de microfísica, de cada evento com tempo de $48 \mathrm{~h}$ de spinup. Posteriormente, foram feitas rodadas intermediárias com ciclos de $3 \mathrm{~h}$ com a finalidade de gerar o background para as rodadas com e sem assimilação dos dados de radar. As rodadas com e sem assimilação foram realizadas com 4 ciclos de 1 hora cada e a integração no tempo (previsão) de todas as rodadas foi de $12 \mathrm{~h}$.

A condição inicial fornecida pela análise do modelo GFS é utilizada somente no primeiro ciclo de $6 \mathrm{~h}$ de assimilação dos dados do GTS (Fig. 2.a). No entanto, a análise do GFS é utilizada como condição de fronteira em

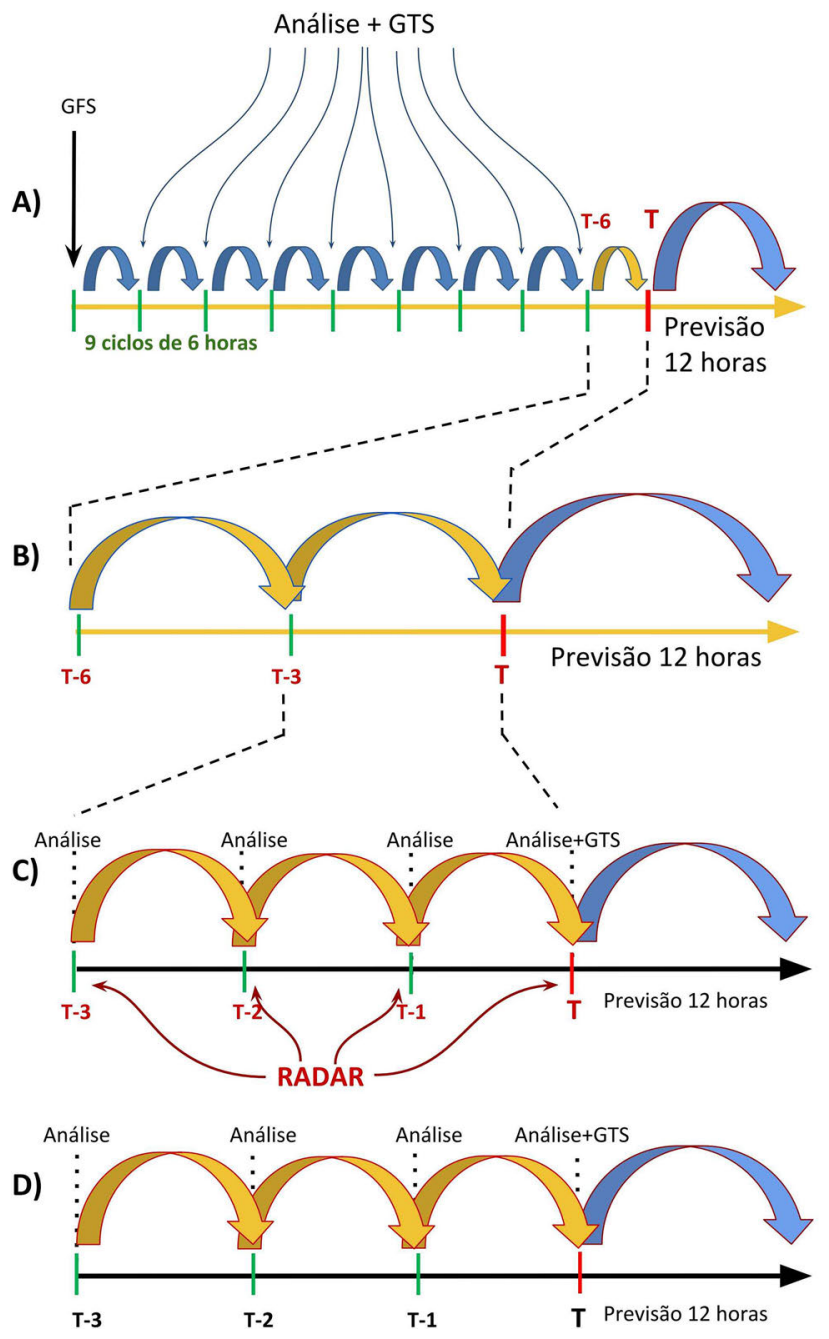

Figura 2 - Ciclo de assimilação de dados e spinup. Em (A) ciclos de assimilação de 6 h com o GFS como condição inicial; (B) ciclos intermediários de 3 h; (C) ciclo horário de assimilação de dados com os dados de radar e em (D) sem os dados de radar. 
todos os demais ciclos de assimilação realizados. A configuração do ciclo de assimilação foi o mesmo utilizado por Vendrasco et al. (2016) e Ferreira et al. (2017), onde a assimilação de dados volumétricos do radar foi inicializada três horas antes do início da previsão (tempo T) para gerar a nova condição inicial para o modelo, e assim integrou no tempo a previsão de 12 h (Fig. 2.b). A descrição das rodadas com e sem assimilação dos dados de radar é demonstrada na Fig. 2.c) e Fig. 2.d), respectivamente.

A avaliação objetiva dos resultados do modelo foi realizada utilizando o índice Fractional Skill Score (FSS), que fornece a fração de acerto do modelo dentro de um limiar de precipitação e de um raio de cobertura especificado (Roberts e Lean, 2008). O FSS possui limitações ao detectar erros na intensidade da precipitação, sendo mais adequado para mostrar a acurácia do posicionamento dos núcleos convectivos na previsão de precipitação para um dado limiar. Além disso, foi aplicado também o Local Root Mean Square Error (LRMSE), utilizado por Vendrasco et al. (2016), que indica a magnitude do erro nos valores de precipitação previstos dentro do mesmo raio de influência que foi utilizado no cálculo do FSS.

Além disso, foi verificado o impacto de cada parametrização na análise utilizando o FSS para verificar onde há um maior acerto na localização da precipitação juntamente com o LRMSE que demonstra a região com menor erro na intensidade dos núcleos convectivos. Desta forma, os dois índices foram aplicados em conjunto para estimar qual configuração de assimilação obteve melhor desempenho em prever a localização e a intensidade de precipitação acumulada para cada rodada do modelo.

$\mathrm{Na}$ validação de precipitação do modelo, foi utilizado o produto Combined Scheme - 3 hours (CoSch3), calculado por Ferreira (2016) a partir da metodologia de Vila et al. (2009) para a inclusão dos dados de precipitação em superfície (estações automática). O CoSch3 é um produto de estimativa de precipitação onde combina-se a estimativa por satélite fornecida pelo Tropical Rainfall Measuring Mission (TRMM) e dados medidos por pluviômetros. Este método proporciona estimativa mais realistas, principalmente em situações onde a estimativa por satélite tem deficiência, como é o caso da precipitação por nuvens quentes (Calheiros, 2013).

\section{Resultados e Discussão}

A partir das rodadas para os três casos citados na Tabela 1 , são mostrados os resultados médios da previsão de precipitação de acordo com as parametrizações microfísicas utilizadas e o impacto da assimilação dos dados de radares na previsão. A subseção 3.1 mostra a avaliação de acordo com a precipitação e a subseção 3.2 mostra um comparativo da refletividade para três horas de previsão do Evento 2 e como as diversas microfísicas se comportam ao representar a refletividade.

\section{1. Índices médios das parametrizações}

A Fig. 3 mostra os valores de FSS para $12 \mathrm{~h}$ de previsão com assimilação de dados de radar e dados convencionais (GTS). As linhas indicam as médias para os três eventos precipitantes analisados, para diferentes limiares. Já a parte sombreada da Fig. indica a flutuação dos valores máximos e mínimos dos índices FSS das parametrizações em todas as rodadas média dos três casos.

Os mais altos valores de FSS para os limiares de $1 \mathrm{e}$ $5 \mathrm{~mm}$ foram usando a ADR para as 6 primeiras horas de previsão conforme mostra a Fig. 3.a) e b), borda superior do sombreada em vermelho. Estes resultados mostram que a condição inicial obtida a partir da assimilação de dados de
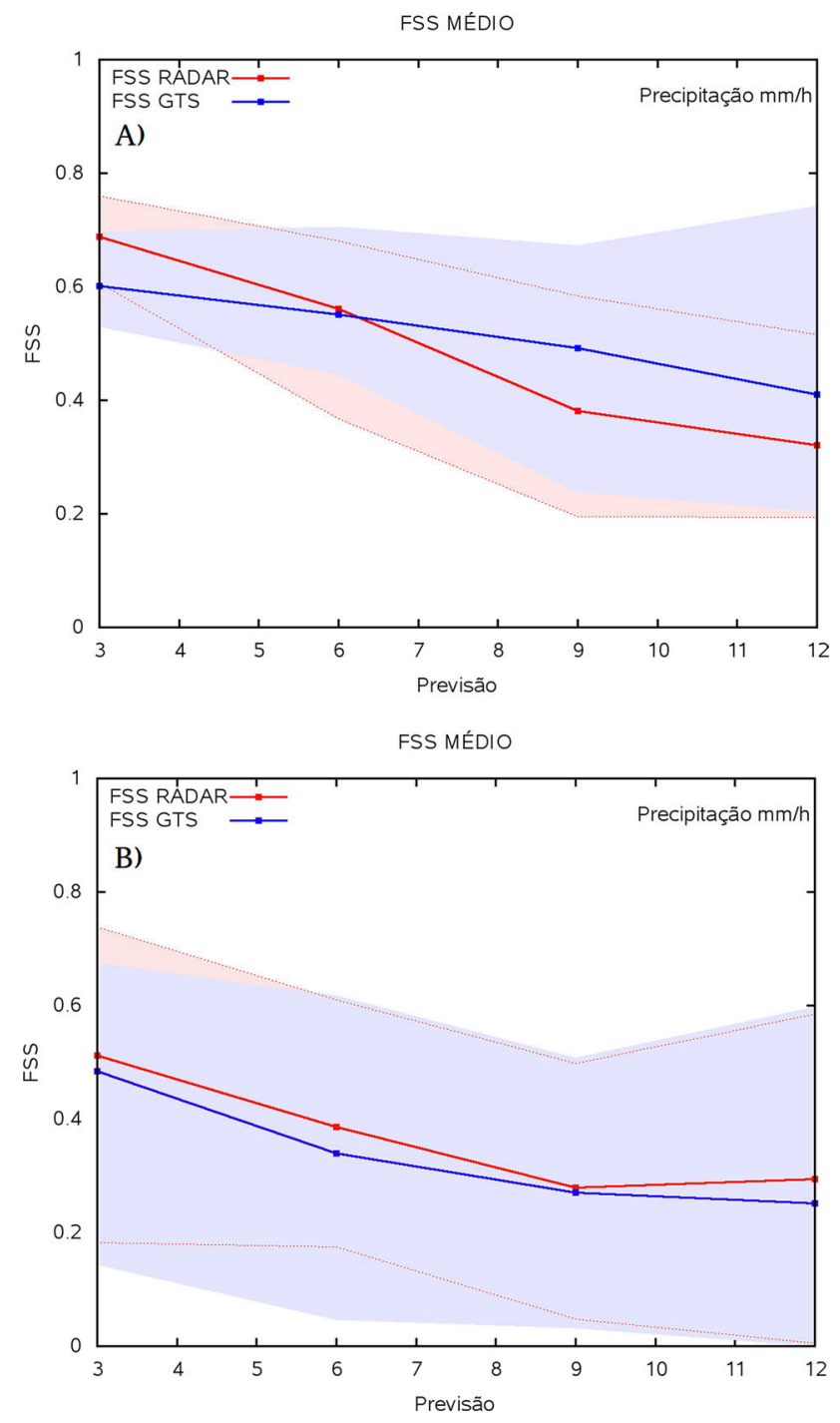

Figura 3 - Comparativo das médias do FSS nos três eventos das 9 parametrizações, onde o sombreado indica a variação entre os máximos e mínimos entre as parametrizações, as linhas correspondem ao comportamento das médias com ADR e sem (GTS). As figuras indicam o FSS médio nos três casos para: (A) limiar de $1 \mathrm{~mm}$ em $10 \mathrm{~km}$ de raio; (B) FSS médio com limite de $5 \mathrm{~mm}$ em $10 \mathrm{~km}$ de raio. 
radar apresenta uma melhor representação da atmosfera para a formação de precipitação nas seis primeiras horas de previsão. Tais resultados concordam com diversos estudos na área de ADR que comprovaram a melhoria na previsão até $6 \mathrm{~h}$ (Ferreira et al., 2017) a $7 \mathrm{~h}$ (Wang et al., 2013).

Para limiares de $5 \mathrm{~mm}$ em um raio de $10 \mathrm{~km}$ (Fig. 3 . b) é possível observar que a média usando ADR é superior a assimilação de dados convencionais (ADC ou gts, nas figuras) durante as $12 \mathrm{~h}$ de previsão.

O ganho com radar (Fig. 3) chega a apresentar na média até $20 \%$ de ganho no FSS, indicada pela distância entre as linhas vermelhas (radar) e azuis (sem radar). A variação média do ganho entre as parametrizações de microfísica chega a 70\% no FSS com a assimilação de dados de radar, como mostra a amplitude do sombreado em vermelho.

A Fig. 4 mostra a taxa de habilidade de acerto de cada parametrização na média dos três eventos. Nas figuras da coluna direita, são os resultados obtidos pela previsão sem assimilação de dados de radar e colunas da esquerda com ADR.

A microfísica de Thompson destaca-se principalmente na localização dos núcleos convectivos com limites acima de $5 \mathrm{~mm}$ de precipitação em $10 \mathrm{~km}$ de raio (Figs. 4.c/d). Esta taxa de acerto da parametrização Thompson é reafirmada no índice LRMSE, indicando também o menor erro na intensidade dos núcleos convectivos nas 6 primeiras horas de previsão. Estes resultados concordam com a pesquisa de Bao et al. (2019), no qual mostraram que Thompson altera mais os campos meteorológicos nas duas primeiras horas de previsão devido ao rápido aumento na precipitação, porém esse crescimento é cessado até $6 \mathrm{~h}$. E que esse comportamento pode levar a instabilidades no modelo no início da previsão.

Já parametrização Morrison apresentou o menor erro (LRMSE) no final das $12 \mathrm{~h}$ de previsão nos casos de menor intensidade da precipitação, e bom desempenho, principalmente para as previsões com ADR, conforme as linhas azuis na Fig. 4 indicam. Já para os casos de maior intensidade de precipitação, foi Thompson que apresentou menor erro, mas diferente de Morrison, é uma parametrização de um momento e que prevê dois momentos apenas para espécies de gelo e chuva. Ainda de acordo com os resultados de Bao et al. (2019), a parametrização de Morrison possui um crescimento na taxa de precipitação mais constante e pode levar a super estimativas ao longo de $6 \mathrm{~h}$ de previsão, pois essa parametrização, apesar de não ter o maior crescimento entre as avaliadas, não cessa o aumento e continua produzindo precipitação ao longo da previsão. Os casos estudados no presente trabalho foram convectivos e geraram acumulados intensos de chuva durante o período de previsão, o que justifica a parametrização de Thompson tido um melhor desempenho nas primeiras horas e Morrison apresentado um bom desempenho principalmente a partir de $6 \mathrm{~h}$.
Dentre todas as microfísicas analisadas, a que obteve o pior desempenho foi a Kessler. Apesar desta parametrização não possuir a representação de fases de gelo e as simulações serem de sistemas convectivos (SC), ela foi inserida para verificar se teriam alterações após a ADR. É possível observar uma melhoria nos valores do FSS até as 6 primeiras horas na previsão ADR utilizando Kessler em relação à previsão contendo apenas os dados convencionais (Figs. 4.b/d e Figs. 4.a/c respectivamente).

$\mathrm{O}$ aumento na magnitude dos erros encontrado no LRMSE a partir de $9 \mathrm{~h}$ de previsão, comparando a assimilação de dados convencionais (ADC) e ADR, deve-se principalmente ao fato da ADR inserir mais umidade ao modelo, podendo gerar convecção espúria em previsões mais longas $(>6 \mathrm{~h})$.

Outro resultado que deve ser notado é o baixo desempenho da parametrização WDM6. Essa parametrização é de dois momentos e mais complexa do que a série de WSM3, WSM5 e WSM6, porém teve desempenho bastante inferior às demais. Estes resultados concordam com os estudos de Van Weverberg et al. (2013), o qual avaliou desempenhos de esquemas de um e dois momentos e verificou que estes nem sempre são superiores aos esquemas de um momento, tendo em vista que a previsão explícita de concentração numérica não necessariamente melhora processos de simulação de nuvem e distribuição de precipitação.

Ressaltando que, a maioria dos trabalhos que avaliam diferentes microfísicas apresentam estudos idealizados de linhas de instabilidade, sistemas convectivos, entre outros. No presente trabalho, as microfísicas são avaliadas em sistemas precipitantes reais, e com alta taxa de precipitação, o que se permite fazer uma analogia aos resultados e compará-los com casos reais.

\subsection{Análise da refletividade}

Para verificar o impacto da assimilação dos dados de radar em função da intensidade da precipitação, os dados foram separados em intervalos de classe de $5 \mathrm{dBZ}$, e verificou-se a quantidade de pontos em cada intervalo gerados pelo modelo - com e sem assimilação dos dados de radar (Fig. 5). Os valores de refletividade podem ser aproximados em taxas de precipitação através de uma relação refletividade-precipitação (relação Z-R), da forma $\mathrm{Z}=\mathrm{aR} \wedge \mathrm{b}$. Diversas relações $Z-R$ existem na literatura, e os coeficientes a e b são dependentes da distribuição de tamanho de gotas (DSD) observada. A relação Z-R mais utilizada na literatura é a relação Marshall-Palmer (Marshall e Palmer, 1948), onde as constantes a e b assumem os valores de 200 e 1,6 respectivamente. A Tabela 3 apresenta os valores de precipitação correspondentes à cada intervalo de classe de refletividade representados no histograma da Fig. 5.

Na Fig. 5 é mostrada a comparação entre a refletividade medida pelo radar ASU e a previsão de refletividade 
A)

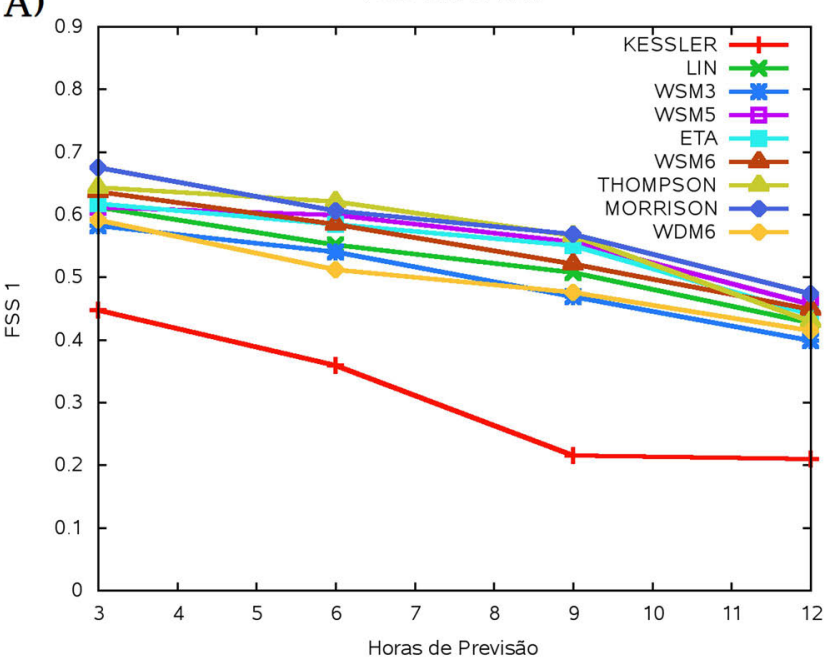

FSS5 SEM RADAR

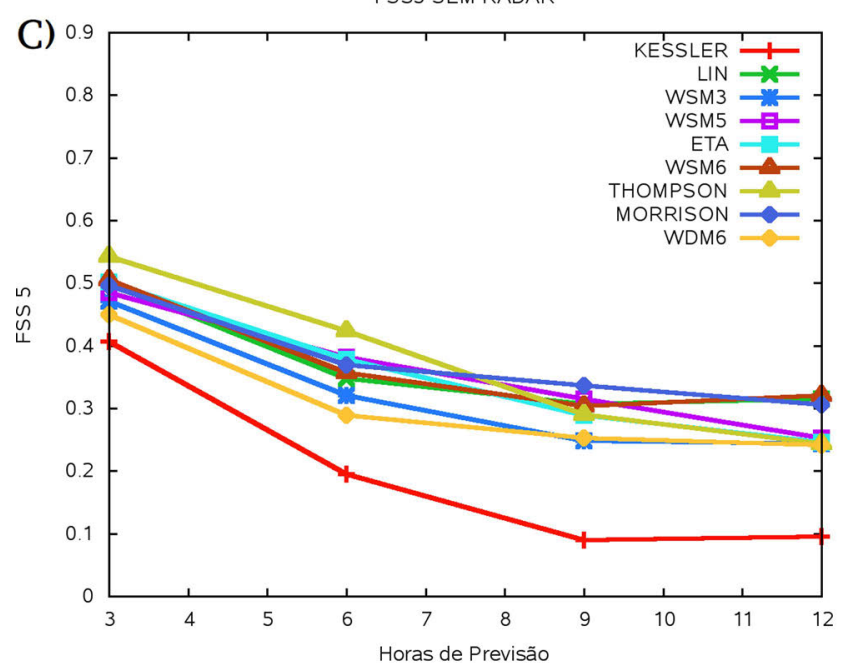

E)

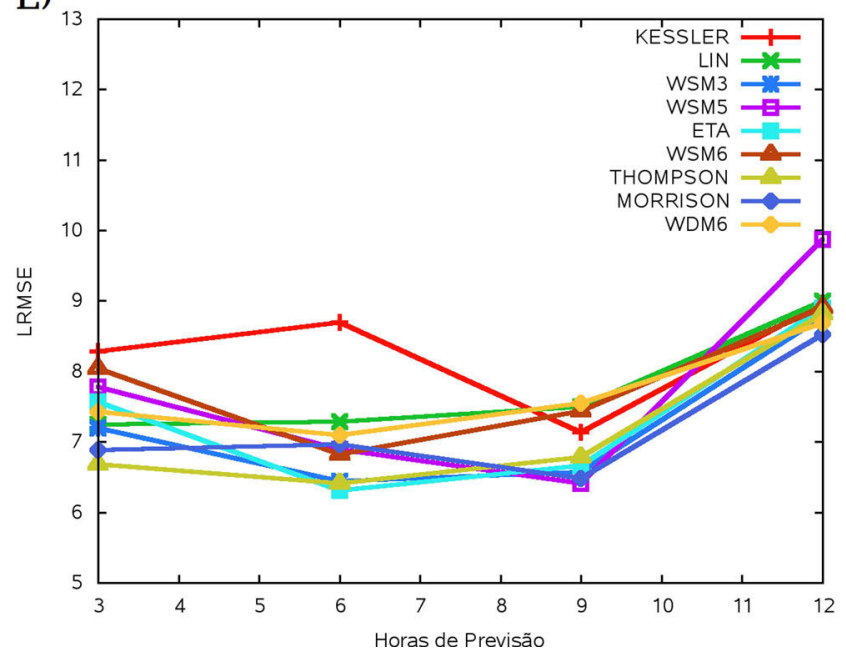

B)

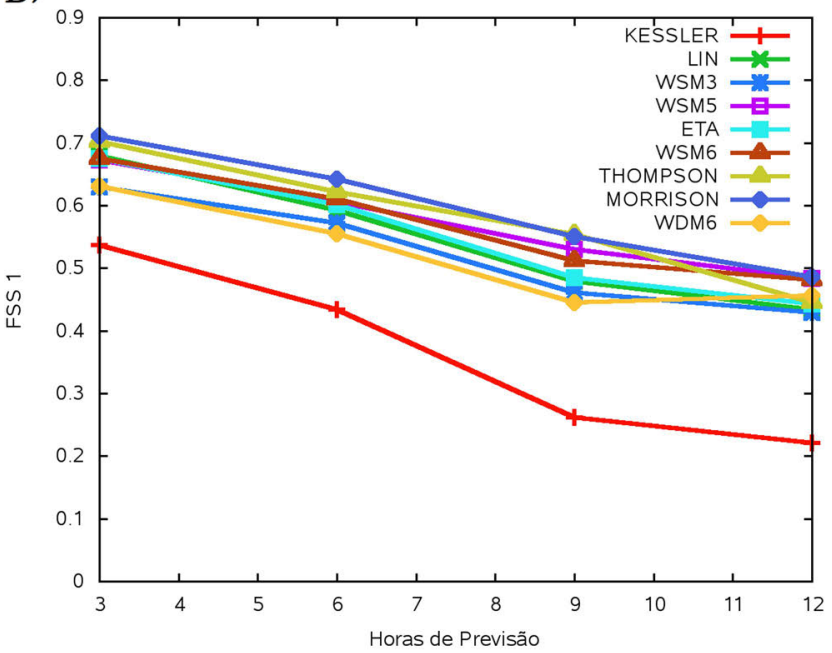

FSS5 COM RADAR
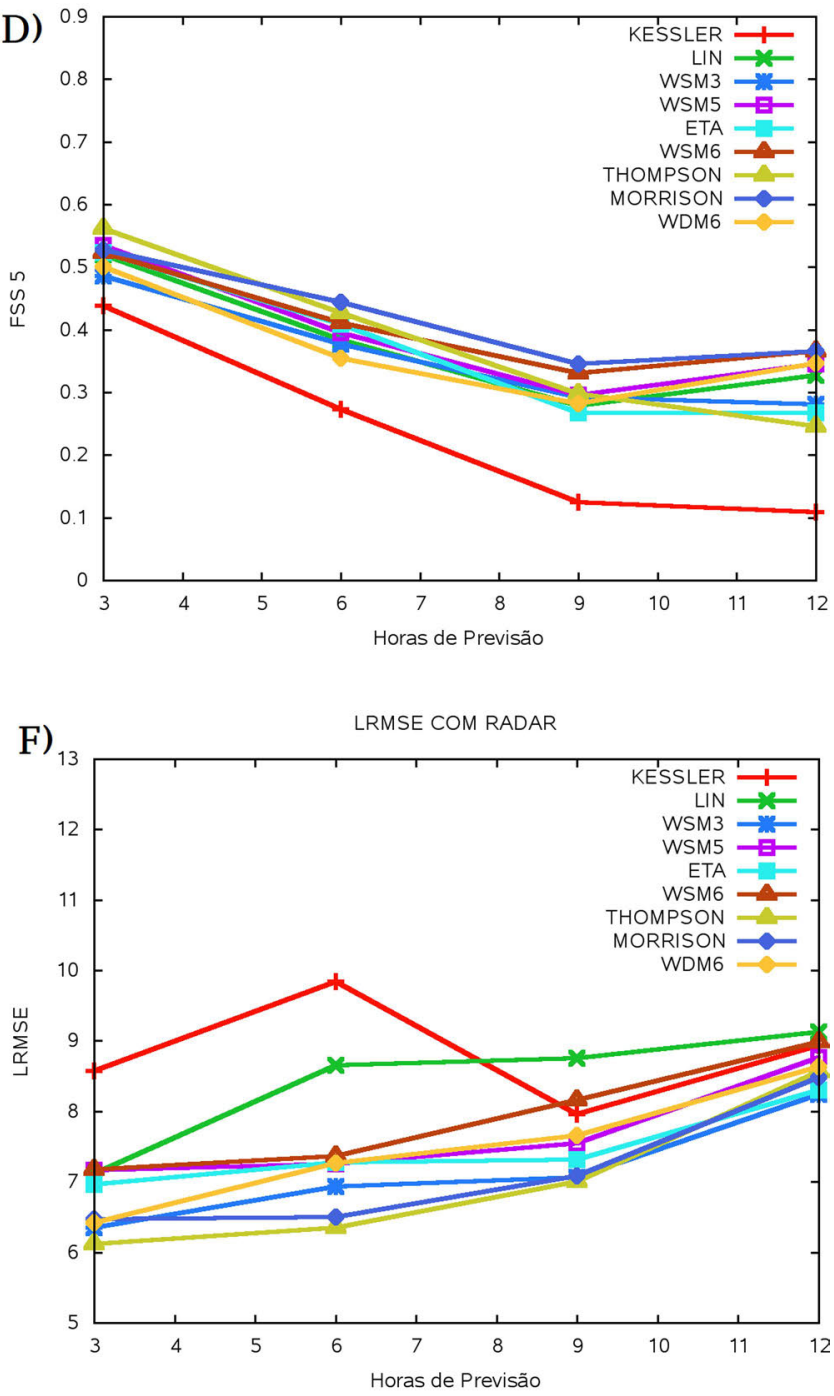

Figura 4 - Comparativo dos índices médios de cada parametrização nos três eventos. FSS com limites de $1 \mathrm{~mm}$ em $10 \mathrm{~km}$ (a) sem assimilação de dados radar, (b) com assimilação de dados radar; FSS com limites de $5 \mathrm{~mm}$ em $10 \mathrm{~km}$ (c) sem assimilação de dados radar (d) com assimilação de dados radar; e LRMSE em $10 \mathrm{~km}$ (e) sem assimilação e (f) com a assimilação de dados radar. 
gerada pelo modelo a partir da CI sem e com assimilação dos dados radar, em Fig. 5.a) e Fig. 5.b) respectivamente. Comparando o comportamento da distribuição dos valores de refletividade previstos pelas diversas microfísicas, nota-se um aumento nos números de pontos de grade que há valores de refletividades - principalmente para os valores mais elevados - quando os dados de radar são assimilados. Observa-se que a ADR fez aumentar o número de parametrizações que superestimaram as ocorrências de precipitação acima de $35 \mathrm{dBZ}(>5,61 \mathrm{~mm} / \mathrm{h}$

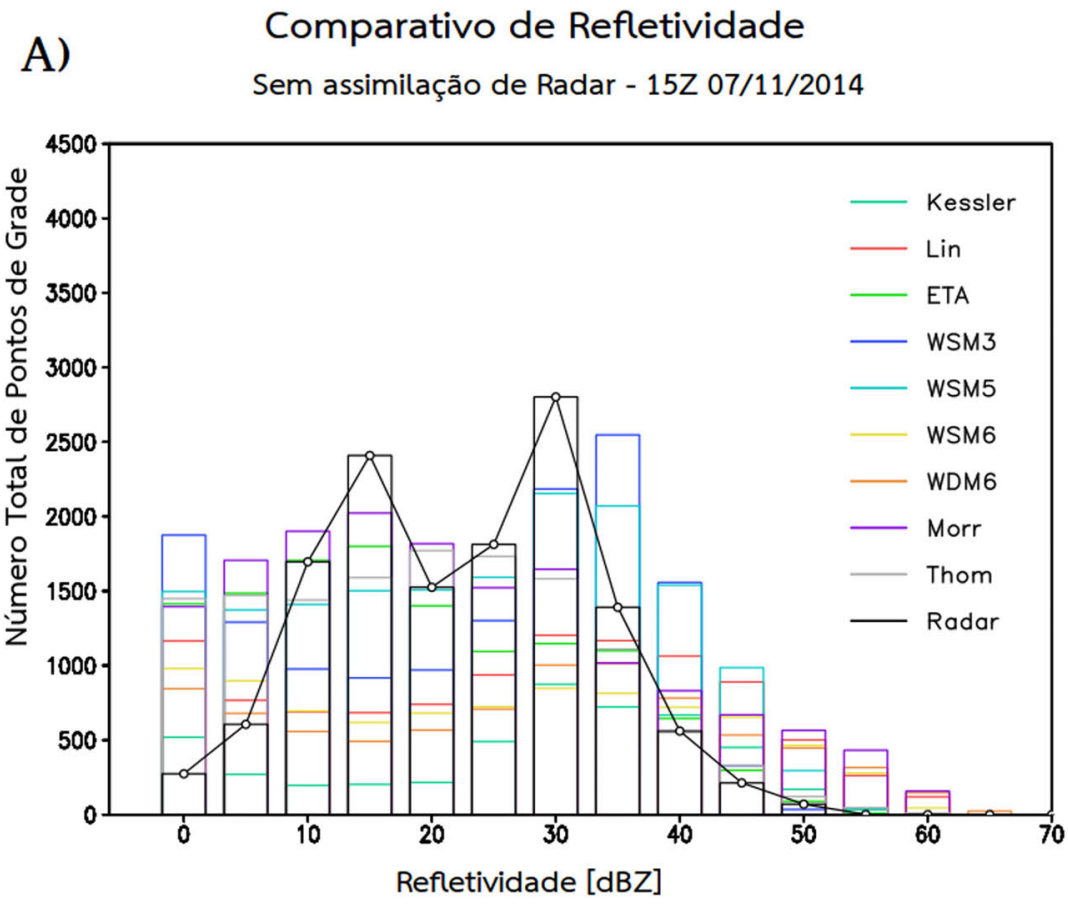

B) Comparativo de Refletividade

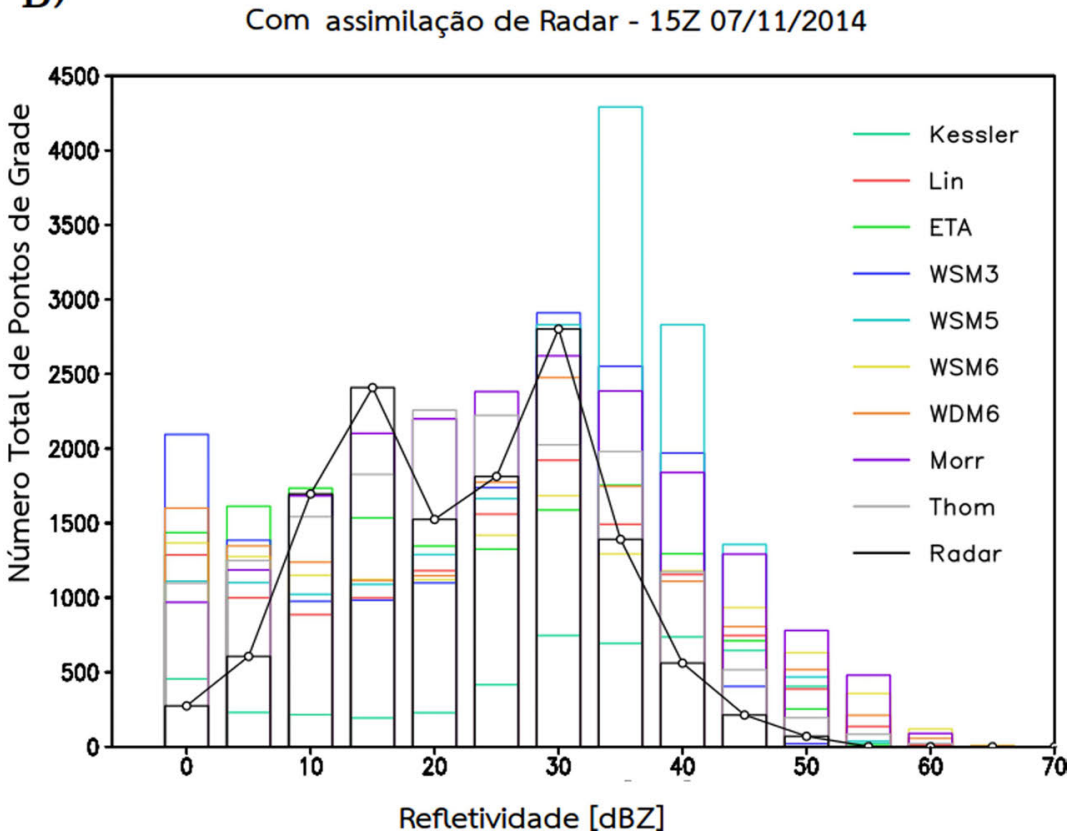

Figura 5 - Comparativo de refletividade do radar e da refletividade gerada pelo modelo em $600 \mathrm{hPa}$ para as $15 \mathrm{Z}$ do dia 07/11/2014 entre todas as parametrizações. Em (A) sem ADR e em (B) com ADR. 
conforme Tabela 3). Isto sugere que modificação da CI no modelo gerada pela ADR representa um estado da atmosfera com mais instabilidade, ao inserir no modelo mais umidade. $\mathrm{Na}$ média das faixas de refletividade a parametrização Morrison manteve-se mais próximo ao dado medido pelo radar de Assunção - Paraguai.

O resultado da parametrização de Kessler não sofreu alteração no número de núcleos convectivos com refletividade entre 25 e $35 \mathrm{dBZ}$ (precipitação moderada) após a assimilação de dados de radar, porém o número de formações convectivas, com valor de refletividade entre 35 e $45 \mathrm{dBZ}$ (precipitação intensa), teve um aumento conforme Fig. 5.b). Esta parametrização, na média dos valores de refletividade, apresentou os resultados mais afastados do observado no radar de ASU, subestimando os valores de refletividade e o número de células de chuva. Isso se deve principalmente ao fato dessa parametrização não ser capaz de representar os processos de gelo (não possui representação de Qi, Qs, Qg).

O Esquema de Lin, após a ADR, apresentou um aumento no número de núcleos entre 20 e 40 dBZ, aproximando-se do observado. Contudo superestimou o número de células convectivas com valores de refletividade acima $35 \mathrm{dBZ}$.

Observa-se que na coluna correspondente a refletividade de $30 \mathrm{dBZ}$, após ADR e exceto o esquema de Kessler, as demais parametrizações aproximaram-se do valor observado, destacando-se as parametrizações WSM5, WSM3, Morrison e WDM6.

A parametrização de Morrison apresentou o melhor desempenho com ADR, mesmo superestimando o número de células convectivas com valores de refletividade acima de 35 dBZ. Já o desempenho da parametrização WSM5 destaca-se com o aumento do número de células com

Tabela 3 - Valores de refletividade e taxa de precipitação a partir da relação Marshall-Palmer.

\begin{tabular}{lc}
\hline Refletividade (dBZ) & Taxa de precipitação $(\mathrm{mm} / \mathrm{h})$ \\
\hline 0 & 0,03 \\
5 & 0,07 \\
10 & 0,15 \\
15 & 0,31 \\
20 & 0,65 \\
25 & 1,33 \\
30 & 2,73 \\
35 & 5,61 \\
40 & 11,5 \\
45 & 23,6 \\
50 & 48,6 \\
55 & 99,8 \\
60 & 205 \\
65 & 421 \\
\hline
\end{tabular}

refletividade entre 35 e $45 \mathrm{dBZ}$, muito acima do observado após a ADR.

\section{Conclusões}

O presente trabalho buscou dar um panorama geral de diversas microfísicas e a forma com que elas respondem na previsão utilizando a análise gerada pela assimilação de dados de radar.

Os resultados obtidos mostraram que os mais altos valores de FSS médio para os três casos foram usando a ADR para as 6 primeiras horas de previsão. Para limiares de precipitação mais intensos foi possível observar que a média usando assimilação de dados de radar foi superior a assimilação de dados convencionais durante as $12 \mathrm{~h}$ de previsão. O ganho com radar chegou a apresentar na média até $20 \%$ de ganho no FSS. Já a variação média do ganho entre as parametrizações de microfísica chegou a 70\% com a assimilação de dados radar.

Dentre as microfísicas avaliadas, a microfísica de Thompson destacou-se principalmente na localização dos núcleos convectivos com limites acima de $5 \mathrm{~mm}$ de precipitação em $10 \mathrm{~km}$ de raio de acordo com a avaliação do índice FSS. O pior resultado encontrado foi a partir da parametrização de Kessler e os melhores resultados foram obtidos usando as microfísicas de Thompson e Morrison.

Observou-se que a assimilação de dados de radar fez as parametrizações superestimarem os núcleos convectivos, de intensa precipitação. Na média das faixas de refletividade a parametrização Morrison manteve-se mais próximo do medido pelo radar.

A escolha de uma microfísica muito simplificada, que não seja capaz de representar as fases do gelo e processos na fase mista não é aconselhada para o estudo de sistemas convectivos (SC). É amplamente reconhecido que os SC possuem gelo no seu interior, devido a grande extensão vertical que estes sistemas atingem. Como sugestão, uma alternativa para melhorar a análise e compreensão das parametrizações de microfísica é o uso de dados de classificação de hidrometeoros que pode ser obtida por dados de radares polarimétricos, usando apenas as microfísicas mais adequadas ao sistema a ser simulado.

\section{Agradecimentos}

Os autores agradecem ao SIMEPAR e à DINAC pela disponibilização dos dados de radar e à Pós Graduação em Meteorologia do INPE.

\section{Referências}

AKSOY, A.; DOWELL, D.C.; SNYDER, C.A. Multi-case comparative assessment of the ensemble Kalman filter for assimilation of radar observations. Part I: Storm-scale ana- 
lyses. Monthly Weather Review, v. 137, n. 6, p. 18051824, 2009.

ARAVÉQUIA, J.A.; SZUNYOGH, I.; FERTIG, E.J.; KALNAY, E.; KUHL, D.; KOSTELICH, E.J. Evaluation of a strategy for the assimilation of satellite radiance observations with the local ensemble transform Kalman filter. Monthly Weather Review, v. 139, n. 6, p. 1932-1951, 2011.

BAO, J.W.; MICHELSON, S.A.; GRELL, E.D. Microphysical process comparison of three microphysics parameterization schemes in the WRF model for an idealized squall line case study. Monthly Weather Review, v. 147, n. 3, p. 30933120, 2019.

CALHEIROS, A.J.P. Propriedades Radiativas e Microfísicas das Nuvens Continentais: Uma Contribuição para a Estimativa da Precipitação de Nuvens Quentes por Satélite. 2013. 180 p. Tese (Doutorado em Meteorologia) Instituto Nacional de Pesquisas Espaciais, São José dos Campos, 2013.

DUDHIA, J. Numerical study of convection observed during the Winter Monsoon Experiment using a mesoscale two-dimensional model. Journal of Atmospheric Sciences, v. 46, n. 20, p. 3077-3107, 1989.

DUDHIA, J., 1996: A multi-layer soil temperature model for MM5. The Sixth PSU/NCAR Mesoscale Model Users' Workshop. Disponível em: http://www2.mmm.ucar.edu/ wrf/users/phys_refs/LAND_SURFACE/5_layer_thermal. pdf

FERREIRA, R.C. Estudo de Sistemas Convectivos de Mesoescala Com Uso de Assimilação de Dados de Radar. Dissertação (Mestrado em Meteorologia) - Instituto Nacional de Pesquisas Espaciais, São José dos Campos, SP, Brasil. 2016.

FERREIRA, R.C.; HERDIES, D.L.; VENDRASCO E.L.; BENETI, C.A.P.; BISCARO, T.S. Impacto da assimilação de dados de radar em sistemas convectivos de mesoescala: Um estudo de caso. Revista Brasileira de Meteorologia, v. 32, n. 3, p. 447-458, 2017.

GAO, J.; XUE, M.; BREWSTER, K.; DROEGEMEIER, K.K. A three-dimensional variational data analysis method with recursive filter for doppler radars. Journal of Atmospheric Oceanic Technology, v. 21, n. 3, p. 457-469, 2007.

HONG, S.Y.; DUDHIA, J.; CHEN, S.H. A revised approach to ice microphysical processes for the bulk parameterization of clouds and precipitation. Monthly Weather Review, v. 132, n. 1, p. 103-120, 2004.

HONG, S.Y.; LIM, JADE, J.O. The WRF single-moment 6-class microphysics scheme (WSM6). Journal of Korean Meteorological Society, v. 42, n. 2, p. 129-151, 2006.

HOUZE JR, R.A. Cloud Dynamics. Academic Press, Seattle, Washington, 2014.

KESSLER, E. Models of microphysical parameters and processes. In: On the Distribution and Continuity of Water Substance in Atmospheric Circulation. Meteorological Monographs, v. 10, p. 26-31, American Meteorological Society, Boston, MA, 1969.

LIM, K.S.S.; HONG S.Y.. Development of an effective doublemoment cloud microphysics scheme with prognostic cloud condensation nuclei $(\mathrm{CCN})$ for weather and climate models. Monthly Weather Review, v. 138, n. 5, p. 1587$1612,2010$.
LIN, Y.L.; FARLEY, R.D.; ORVILLE, H.D. Bulk parameterization of the snow field in a cloud model. Journal of Climate and Applied Meteorology, v. 22, n. 6, p. 1065-1092, 1983.

MARSHALL, J.S., PALMER, W.M. The distribution of raindrops with size. Journal of Meteorology, v. 5, n. 4, p. 165166, 1948.

MLAWER, E.J., TAUBMAN, J.S., BROWN, D.P., IACOMO, M.J., CLOUGH, S.A. Radiative transfer for inhomogeneous atmospheres: RRTM, a validated correlated-K model for the longwave. Journal of Geophysical Research, v. 102, n. D14, p. 16663-16682, 1997.

MORRISON, H.; THOMPSON, G.; TATARSKII, V. Impact of cloud microphysics on the development of trailing stratiform precipitation in a simulated squall line: Comparison of one-and two-moment schemes. Monthly Weather Review, v. 137, n. 3, p. 991-1007, 2009.

REICHLE, R.H. Data assimilation methods in the Earth Sciences. Advances in Water Resources, v. 31, n. 11, p. 1411-1418, 2008.

ROBERTS, N.M.; LEAN, H.W. Scale selective Verification of rainfall accumulations from high-resolution forecasts of convective events. Monthly Weather Review, v. 136, n. 1, p. 78-97, 2008.

SKAMAROCK, W.C.; KLEMP, J. B.; DUDHIA, J.; GILL, D. O.; BARKER, D.M.; DUDA, M.G.; HUANG, X.Y.; WANG, W.; POWERS, J.G. A description of the advanced research WRF version 3. NCAR Technical Note-475 +STR, 113 pp. 2008.

STENSRUD, D.J.; BAO, J.W.; WARNER, T.T. Using initial condition and model physics perturbations in short_range ensemble simulations of mesoscale convective systems. Monthly Weather Review. v. 128, n. 7 p. 2077-2107. 2000.

SUN, J.; CROOK, N.A. Dynamical and microphysical retrieval from doppler radar observations using a cloud model and its adjoint: I. model development and simulated data experiments. Journal of the Atmospheric Sciences, v. 54, n. 12. p. 1642-1661, 1997.

THOMPSON, G.; FIELD, P.R.; RASMUSSEN, R.M.; HALL, W.D. Explicit forecasts of winter precipitation using an improved bulk microphysics scheme. Part II: Implementation of a new snow parameterization. Monthly Weather Review, v. 136, n. 12, p. 5095-5115, 2008.

WEVERBERG, K.V.; VOGELMANN, A.M.; LIN, W.; LUKE, E.P.; CIALELLA, A.; MINNIS, P.; KHAIYER, M.; BOER, E.R.; JENSEN, M.P. The role of cloud microphysics parameterization in the simulation of mesoscale convective system clouds and precipitation in the tropical western Pacific. Journal of the Atmospheric Sciences, v. 70, n. 4, p. 1104-1128, 2013.

VENDRASCO, E.P.; SUN, J.; HERDIES, D. L.; ANGELIS, F.C. Constraining a 3DVAR radar data assimilation system with large-scale analysis to improve short-range precipitation forecasts. Journal of Applied Meteorology and Climatology, v. 55, n. 3, p. 673-690, 2016.

VENDRASCO, E.P.; SUN, J.; WANG, H.; HERDIES, D.L.; ANGELIS, C.F.; ALBRECHT, R.I. Assimilation of the CHUVA Project's dual polarization radar data with WRF $3 \mathrm{D}-\mathrm{VAR}$ and its impact on the short-range precipitation 
forecast. In: Proc. 36 Conference On Radar Meteorology, 2013, Breckenridge, Colorado. 2013. DVD

VILA, D.A.; GONÇALVES, L.G.G.; TOLL, D.L.; ROZANTE, J.R. Statistical evaluation of combined daily gauge observations and rainfall satellite estimates over continental South America. Journal of Hydrometeorology, v. 10, n. 2, p. 533-543, 2009.

WANG, H.; SUN, J.; FAN, S.; HUANG, X.Y. Indirect assimilation of radar reflectivity with WRF 3D-VAR and its impact on prediction of four summertime convective events. Journal of Applied Meteorology and Climatology, v. 52, n. 4, p. 889-902, 2013.

WARNER, T.T.. Numerical Weather and Climate Prediction. University Press, Cambridge, 2010.
XIAO, Q.; SUN, J. Multiple-radar data assimilation and shortrange quantitative precipitation forecasting of a squall line observed during IHOP 2002. Monthly Weather Review, v. 135, n. 10, p. 3381-3404, 2007.

ZHANG, S.Q.; ZUPANSKI, M.; HOU A.Y.; LIN, X.. CHEUNG, S. H. Assimilation of precipitation affected radiances in a cloud resolving wrf ensemble data assimilation system. Monthly Weather Review, v. 141, n. 2, p. 754-772, 2013.

License information: This is an open-access article distributed under the terms of the Creative Commons Attribution License (type CC-BY), which permits unrestricted use, distribution and reproduction in any medium, provided the original article is properly cited. 